\title{
Compact Non-Invasive Millimeter-Wave Glucose Sensor
}

\author{
Peter H. Siegel ${ }^{1}$, Adrian Tang ${ }^{2}$, Gabriel Virbila ${ }^{3}$, Yanghyo Kim ${ }^{3}$, M. C. Frank Chang ${ }^{4}$ and Victor Pikov ${ }^{5}$ \\ ${ }^{1}$ California Institute of Technology and THz Global, Pasadena, CA, USA \\ ${ }^{2}$ UCLA and NASA Jet Propulsion Laboratory, Los Angeles, CA, USA \\ ${ }^{3}$ UCLA, Los Angeles, CA USA \\ ${ }^{4}$ UCLA and National Chiao Tung University, Hsinchu, Taiwan \\ ${ }^{5}$ Glaxo Smith Kline, Stevenage, UK
}

\begin{abstract}
The authors describe a compact non-invasive CMOS-circuit-based glucose monitor using millimeter-wave transmission for use on animal and human subjects. Using an earlier device, in vivo measurements were performed through the ear in anesthetized animals and correlated with blood glucose concentration from test strips. In addition, millimeter wave absorption through glucose-containing solutions was measured in specialized liquid transmission cells and is shown to correlate with the animal and separate in vitro data. Design and performance information on the CMOS transceiver are given.
\end{abstract}

\section{INTRODUCTION}

C

ONTINUOUS NON-INVASIVE monitoring of blood glucose levels is a holy grail for researchers working on type 2 diabetes mellitus, estimated to affect almost 300 million people worldwide [1]. In 2014, two of the authors showed that millimeter-wave (MMW) transmission at a frequency between 27 and $40 \mathrm{GHz}$ (Ka band) can be directly correlated with absolute readings of blood glucose levels in anesthetized rats [2]. MMW transmission through the ear reproducibly tracked changes in glucose levels (measured by blood glucose test strips) following injections of dextrose and insulin, and showed no change upon injections of saline (Fig. 1).

The positive correlation between blood glucose levels and MMW transmission through the rat ear, coupled with signal changes that were well above the system noise floor when transmitter power is in the allowable safe exposure levels $\left(1 \mathrm{~mW} / \mathrm{cm}^{2}\right.$ for six minutes [3]), are very promising, but the rigid waveguide hardware used in the original experiments can only be employed on immobilized animals. The authors have now designed and fabricated a small light-weight clamp-on fixture containing a full MMW CMOS transceiver chip set and input/output antenna coupled to a tethered power/data transfer cable for active animal and human studies. Laboratory measurements were also conducted on the transmission of MMWs through solutions of saline, and saline plus sugar, to see if they are consistent with both the in vivo rat ear measurements and in vitro measurements on blood made by other researchers. This paper discusses the in vitro and in vivo measurements, the CMOS transceiver design, and the design for a packaged noninvasive glucose monitoring device.

\section{IN VITRO AND IN VIVO EXPERIMENTS}

We have shown [2] that low levels of transmitted MMW power through the ear of an anesthetized rat may be conveniently monitored and correlated with changes in the levels of blood glucose in vivo. The exact mechanism for these observed changes in MMW absorption is as yet uncertain, but

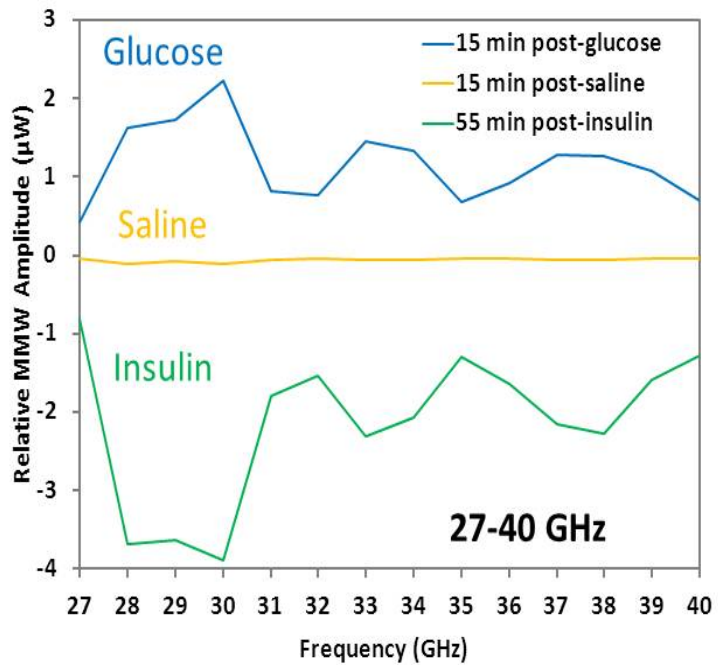

Fig.1. Measured changes in MMW transmission through rat ear after injections of $1 \mathrm{gm} / \mathrm{kg}$ glucose, $5 \mathrm{ml}$ saline (control) and $2 \mathrm{U} / \mathrm{kg}$ insulin [2]. The power levels are extrapolated from the measured square law detector responsivity (approximately $0.28 \mathrm{mV} / \mu \mathrm{W}$ ).

the effect is quite pronounced. In our in vivo measurements we observed a strong reduction in MMW power absorption through the rat ear with increasing glucose levels in the blood. This effect has also been observed in in vitro blood samples [4], [5]. In an attempt to quantify whether or not this change is due to a metabolic process (e.g. glycolysis) or to glucose concentration directly, we performed a series of laboratory absorption measurements for glucose and other fluids.

Using a vector network analyzer (Anritsu 37397C) with our $\mathrm{Ka}$ band waveguide magnetically clamped around a sealed $10 \mathrm{ml}$ fluid chamber (OptiCell, Thermo Fisher Scientific, Rochester, NY) [Fig. 2 insert], we recorded the transmitted power as a function of frequency for the following fluids: DI water, saline $(0.9 \mathrm{gm} / \mathrm{dl})$ and saline plus glucose $(1 \mathrm{gm} / \mathrm{dl})$. The results are shown in Fig. 2, where it is clear that adding glucose to saline reduced the absorbed MMW power in the solution, just as in both the in vivo [2] and in vitro blood measurements [4], [5] made by other researchers. Note that the data for glucose plus saline represents a concentration 10X a typical level in vivo and accounts for the much greater increase in transmission than what we observed in the rat studies (red vs blue in Fig. 2). We also were able to find a supporting article [6], which yielded similar results on saline plus glucose solutions with concentrations comparable to those observed in vivo. Although not conclusive, these measurements strengthen the case for being able to directly measure glucose concentration, rather than some indirect glucose-triggered effect in vivo. 


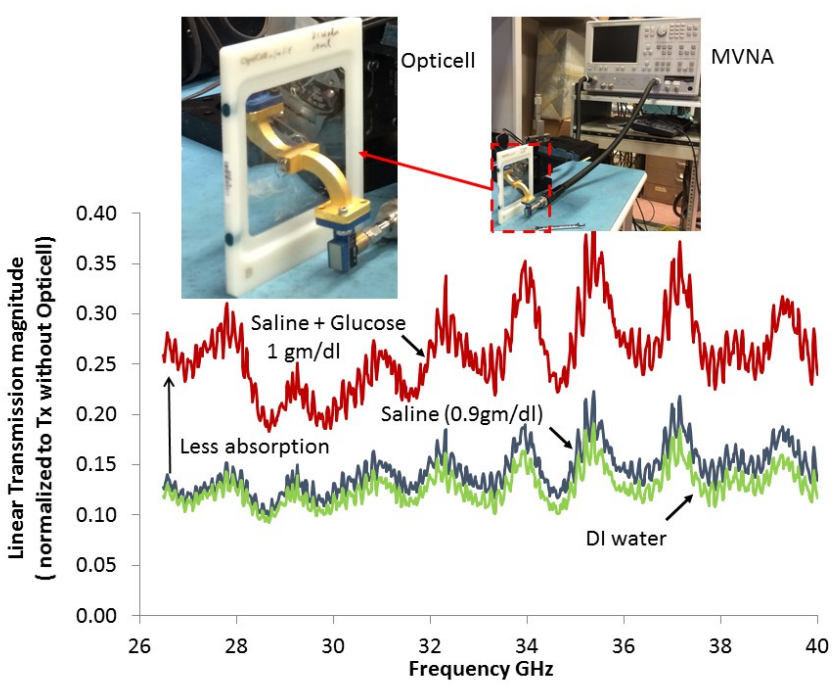

Fig. 2. RF transmission vs. frequency normalized to transmission without Opticell for DI water (green), saline (blue) and glucose plus saline (green). $1.0=$ no loss, $0.0=$ zero transmission. Incident power $=$ $1-1.3 \mu \mathrm{W}$. Transmitted power $=0.05-0.1 \mu \mathrm{W}$. Peaks and valleys are due to reflection off the Opticell.

\section{CMOS CHIP SET}

The in vivo and in vitro measurements correlating glucose concentration increase to a reduction in MMW absorption, spurred us to design and fabricate a compact CMOS RFIC transceiver chip set that could be assembled into a wearable clip-on package, and used on awake and freely moving animals or humans. The chipset has the following functional elements: a tunable free running MMW oscillator, a power amplifier, a non-coherent pre-amplified receiver, and frequency and power control circuitry. DC power, detected signals, and all control functions on the RFIC are introduced via a 10 pin input/output connector and cable tethered to a control computer USB port. Future RFIC generations will use wireless input/output for control and data transfer. The chips were laid out and fabricated in a standard $65 \mathrm{~nm}$ foundry process from TSMC, Taiwan and are shown in Fig. 3.
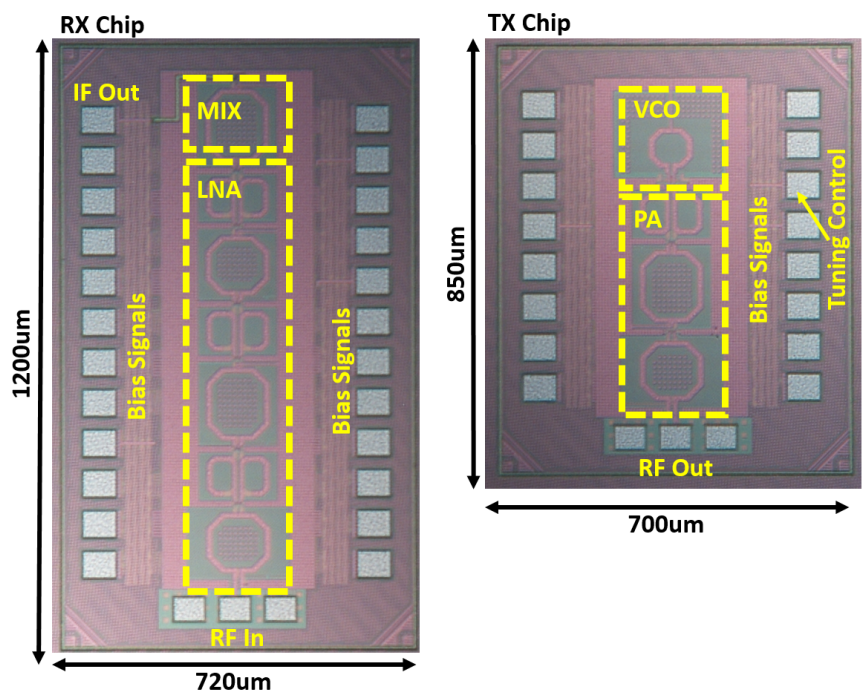

Fig.3. Die photographs of both the transmitter and receiver chips with overall dimensions in microns.
The transmitter is designed to generate up $3.1 \mathrm{~mW}$ of tunable RF CW energy between 33 and $37 \mathrm{GHz}$. The receiver uses a non-coherent self-mixing detection circuit with an input referred detection limit of 1 nanowatt $(-60 \mathrm{dBm})$. The expected loss through the tissue is between 20 and $30 \mathrm{~dB}$, leaving plenty of detection headroom even at $1 \mathrm{~mW} / \mathrm{cm}^{2}$ input power density providing a final signal to noise $(\mathrm{S} / \mathrm{N})$ of $30-40 \mathrm{~dB}$. When higher losses are encountered the gain can be increased substantially by reducing the transmitter duty cycle without exceeding any SAR safe limits in the tissue. Modulation is applied to the transceiver through a standard TTL input port.

\section{Packaged Transceiver}

The transmit and receive chips are integrated onto the ends of separate printed circuit boards (PCB) composed of a $1.5 \mathrm{~mm}$ thick RO4350B/bondply sandwich. Appropriate dielectric and metal ground plane regions are pre-etched into the PCB. Each RFIC must be coupled to an RF antenna that can radiate into the tissue (transmitting antenna) and pick up the power on the other side (receiving antenna). A small folded dipole was designed and placed on the PCB close to the RFICs and coupled to the output/input port via short wire bonds (Fig. 4).

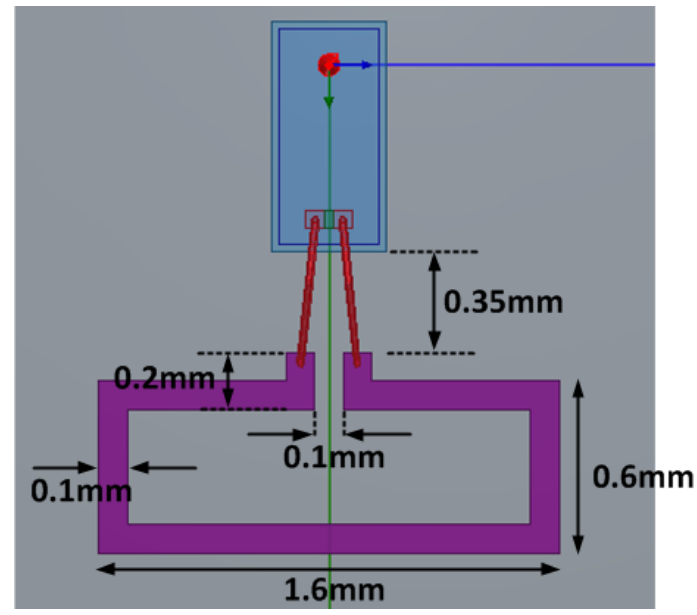

Fig. 4.Antenna design for both transmitter and receiver.

The power, data and control tabs on each RFIC are wire bonded to pads that lead to a small 10 pin connector on the opposite end of the PCB. The antenna and RFICS are then coated with a BCB protection layer (Dow Chemical). Matching to the tissue is accomplished by sanding the BCB layer down to a flat half-wavelength thick sheet and then adding (gluing) a quarter wavelength thick matching layer composed of low loss glass on top. A diagram of the antenna structure and EM simulation results using HFSS showing the predicted power transfer characteristics through $1 \mathrm{~mm}$ of tissue are given in Figs. 5 and 6.

The two PCBs are tied together in a clothes-pin type of arrangement using rubber bands and a small cylinder as a pivot for the clamp (Fig. 7). Once in place the antennas are in intimate contact with the tissue on both the transmitter and receiver sides, and continuous measurements can be performed on either animals or humans while the subjects are in an active state. The only inconvenience is the wire tether, which as stated earlier, will be replaced by a battery and wireless transceiver system in the next generation circuitry. 


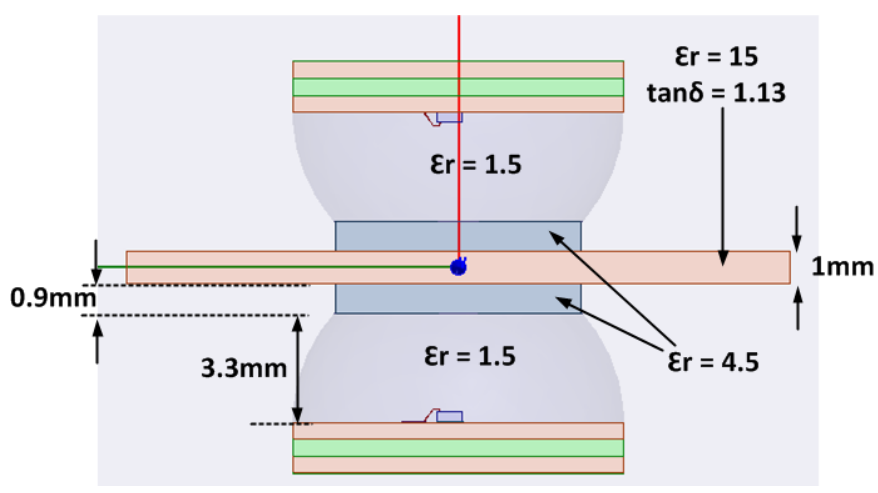

Fig. 5. HFSS layout for transmitting and receiving antennas separated by $1 \mathrm{~mm}$ of tissue (rat ear simulant).
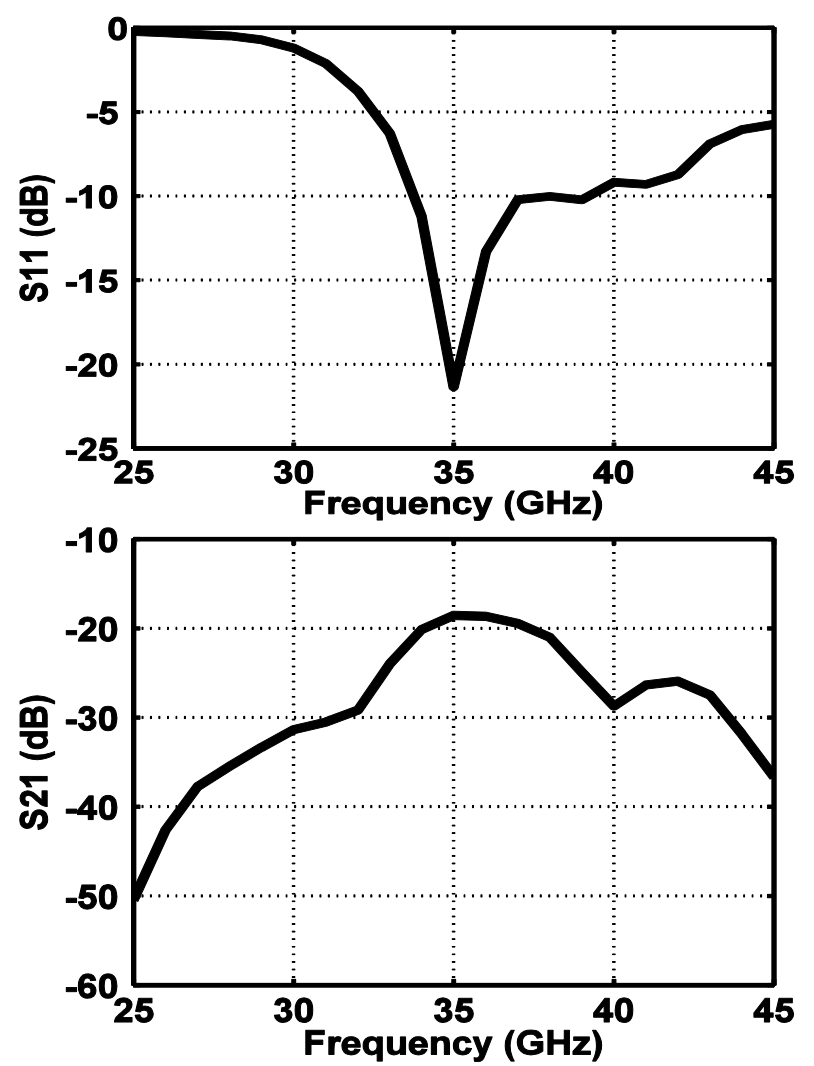

Fig. 6. Simulated (HFSS) transmitter matching (top) and power transfer function from transmit to receive antenna (bottom) for layout shown in Fig. 5.

At time of writing the chips have been fabricated and delivered and the PCB holders have been sent out for processing. We expect to put the measurement clamp together and begin programming within the month. The first tests will be on awake rats with human trials to follow.

\section{SUMMARY}

After initial successful experiments tracking blood glucose levels via MMW transmission through the ears of anesthetized rats, and accurately correlating MMW absorption with separate blood glucose test strips, the authors have been encouraged to extend their initial work. In this short paper they present in vitro absorption measurements on solutions of saline and saline plus glucose, which correlate in direction and magnitude with absorption changes measured in vivo. They also present a CMOS RFIC transceiver chipset operating between 33 and $37 \mathrm{GHz}$ that has the capacity to make high sensitivity coherent transmission or reflection measurements in $\mathrm{CW}$ or variable-duty-cycle pulsed operation. The transmitter and receiver modules have been coupled to a custom designed planar antenna that has been fabricated on a separate PCB board that also serves as a holder and clamping device for in vivo measurements. The CMOS chips and antennas have been covered with a $\mathrm{BCB}$ protection layer that has been machined down to accept a matching layer that will help provide better power transfer into and out of the subject tissue. Designs and numeric simulations for the transceiver performance are presented.

A simple clothes-pin style mount has been realized with a very flexible, but tethered input/output wired control system. Software for controlling the measurements and collecting the glucose data has yet to be developed. Since the instrumentation is designed to measure changing glucose levels, rather than absolute concentrations, we envision a continuously updated, individually calibrated system for each user that can both establish a long term baseline for every activity level and warn of dramatic change without having to continuously calibrate against blood sampling. The clamp-on device will be tested on awake and active animals, and eventually configured for human trials.

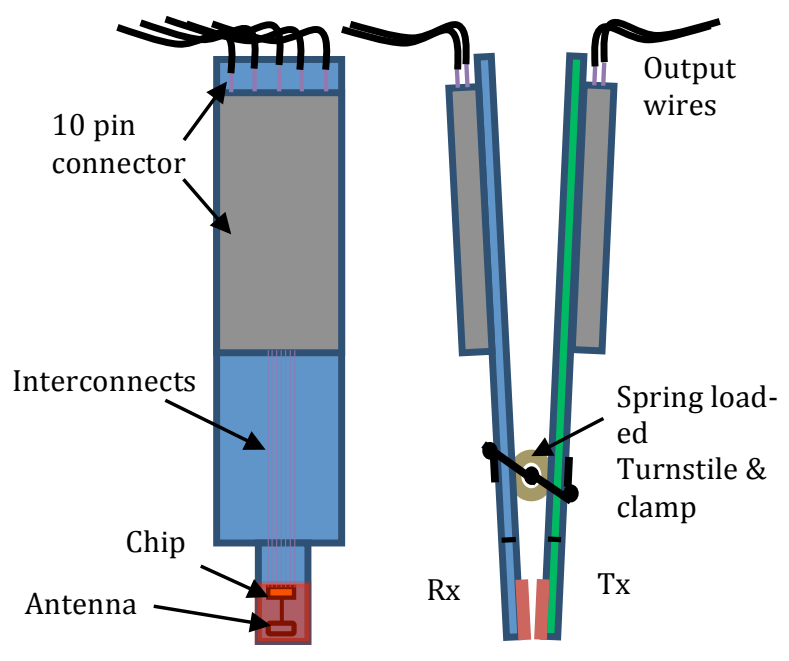

Fig. 7. .Layout for final clothes pin style clamp.

\section{REFERENCES}

[1]. Lei Chen, Dianna J. Magliano, and Paul Z. Zimmet, "The worldwide epidemiology of type 2 diabetes mellitus - present and future perspectives," Nature Reviews Endocrinology, vol. 8, pp. 228-36, 2012.

[2]. P. H. Siegel, Y. Lee and V. Pikov, "Millimeter-wave non-invasive monitoring of glucose in anesthetized rats," $39^{\text {th }}$ Int. Conf. on Infrared, Millimeter, and THz Waves, paper T2_D8.10, Tucson, AZ, USA, Sept. 14-19 $9^{\text {th }}, 2014$.

[3]. IEEE Committee on Electromagnetic Safety (SCC39), "IEEE Standard for Safety Levels With Respect to Human Exposure to Radio Frequency Electromagnetic Fields, $3 \mathrm{kHz}$ to $300 \mathrm{GHz}$," Piscataway, NJ: IEEE, c. 2006.

[4]. M. Hofmann, G. Fischer, R. Weigel and D. Kissinger, "Microwave-Based Noninvasive Concentration Measurements for Biomedical Applications," IEEE Trans. MTT, v. 61, no. 5, 2195-2202, 2013.

[5]. Tutku Karacolak, Elaine C. Moreland, and Erdem Topsakal, "Cole-Cole Model for Glucose Dependent Dielectric Properties of Blood Plasma for Continuous Glucose Monitoring," Microwave and Optical Tech. Lett., v.55, no.5, 116-64, 2013.

[6]. Y. Nikawa and D. Someya. "Non-invasive measurement of blood sugar level by millimeter waves," IEEE Int. Mic. Sym. Digest, pp. 171-4, 2001. 